\title{
Optics manufacturing research projects by undergraduates who happen to be women
}

Stephen Jacobs, Leslie Gregg, Edward Fess, John Schoen

Stephen D. Jacobs, Leslie L. Gregg, Edward M. Fess, John M. Schoen, "Optics manufacturing research projects by undergraduates who happen to be women," Proc. SPIE 9663, Eighth International Topical Meeting on Education and Training in Optics and Photonics, 96632D (6 October 2003); doi: $10.1117 / 12.2208550$

Event: Eighth International Topical Meeting on Education and Training in Optics and Photonics, 2003, Tucson, Arizona, United States 


\title{
Optics manufacturing research projects by undergraduates who happen to be women
}

\author{
Stephen D. Jacobs, Leslie L. Gregg, Edward M. Fess, and John M. Schoen \\ University of Rochester, 240 East River Road, Rochester, New York 14623 \\ telephone: 585-275-2478; fax: 585-275-7225; e-mail:sjac@lle.rochester.edu
}

\begin{abstract}
Significant research is conducted during the school year and over summer months at the Center for Optics Manufacturing (COM) by undergraduates. Projects are designed with short- and long-term goals, to be carried out by students using advanced $\mathrm{CNC}$ machines and sophisticated metrology tools. Five activities are described with emphasis on the individuals doing the work.

(C)2003 Optical Society of America

OCIS codes: (220.4610) Optical fabrication; (220.5450) Polishing
\end{abstract}

\section{Summary}

Research projects are conducted at the Center for Optics Manufacturing (COM) by undergraduate students from the University of Rochester and Monroe Community College. These projects are designed to expose the students to novel manufacturing technologies, new $\mathrm{CNC}$ machines, and the latest metrology instrumentation. This paper describes the experiences of five students, each of whom has worked at COM (part- and full-time) over the past two to three years. They assembled and presented the significant results of their five projects in poster papers at SPIE's Optifab 2003 in Rochester, NY, on May 19-22, 2003.

Optical Polishing Pitch: Our undergraduates have been compiling a data base of physical properties of optical polishing pitches for two years. Rupal Varshneya ('05 U of R Optics major) conducted a set of experiments for a commercial vendor to evaluate the company's experimental product in comparison with accepted standard products whose properties are tabulated in our data base.

Grain Decoration in ALON: Aluminum oxynitride is a very hard polycrystalline ceramic that is difficult to polish. Anne Marino ('05 U of R Mathematics major) expanded upon developed protocols for studying the decoration of grain structure in ALON disks during polishing with custom synthesized, bound abrasive lapping disks.

Bound Abrasive Smoothing on a CNC Platform: Removal of tool marks from a CNC generated glass part would reduce the time needed for polishing. Adrienne Schoeffler ('03 Monroe Community College in Optical Technology) manufactured and evaluated the smoothing performance for a variety of bound abrasive compositions used as contour tools on a commercial CNC grinding platform.

Visualization of Stresses in Optics: It is important to quantify stress-birefringence in polarization optics from the manufacturing stage through installation and use. Jennifer Sternal ('05 U of R Nursing major) employed a new commercial birefringence mapper to make detailed retardance maps of parts that included laser glass samples, laser damaged glass, liquid crystal devices, birefringent crystals, molded polymers, and a light-weighted mirror assembly.

Magnetorheological Finishing of PMMA: Magnetorheological finishing (MRF) is a novel process that exerts no normal load on the work piece during $\mathrm{CNC}$ polishing. It should be suitable for polishing of soft, diamond turned polymer optics that are nearly impossible to finish conventionally. Starting early in her senior year, Jessica DeGroote ('02 U of R Optics and current Optics PhD student) developed and successfully demonstrated a new magnetorheological (MR) fluid for finishing disks of PMMA. 